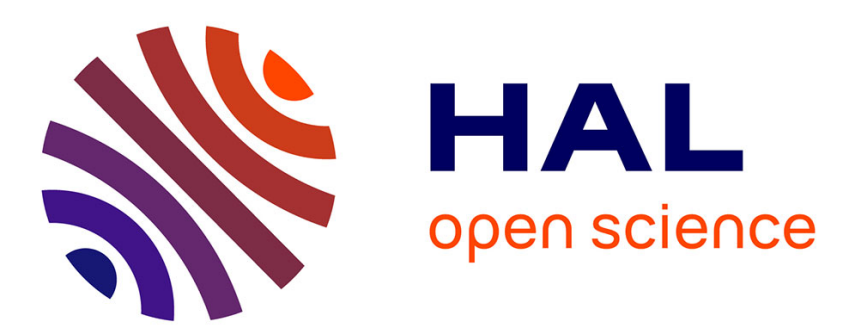

\title{
L'influence des CHSCT sur le bien-être des salariés et sur les accidents du travail. Une étude exploratoire Gregor Bouville
}

\section{To cite this version:}

Gregor Bouville. L'influence des CHSCT sur le bien-être des salariés et sur les accidents du travail. Une étude exploratoire. Revue de Gestion des Ressources Humaines, 2016, 101 (3), pp.24-42. 10.3917/grhu.101.0025 . hal-03227029

\section{HAL Id: hal-03227029 \\ https://hal.science/hal-03227029}

Submitted on 16 May 2021

HAL is a multi-disciplinary open access archive for the deposit and dissemination of scientific research documents, whether they are published or not. The documents may come from teaching and research institutions in France or abroad, or from public or private research centers.
L'archive ouverte pluridisciplinaire HAL, est destinée au dépôt et à la diffusion de documents scientifiques de niveau recherche, publiés ou non, émanant des établissements d'enseignement et de recherche français ou étrangers, des laboratoires publics ou privés. 


\title{
L'influence des CHSCT sur le bien-être des salariés et sur les accidents du travail. Une étude exploratoire
}

\author{
Gregor Bouville \\ Université Paris-Dauphine, PSL Research University \\ CNRS, UMR [7088], DRM, [Management et Organisation] \\ 75016 PARIS, France
}

\begin{abstract}
This is a pre-publication version of:
Bouville G. (2016), 'L'influence des CHSCT sur le bien-être des salariés et sur les accidents du travail. Une étude exploratoire', Revue de Gestion des Ressources
\end{abstract}

Humaines, $\mathrm{n}^{\circ}$ 101, p. 24-42

\section{Résumé:}

La présence d'une instance représentative du personnel en charge de la santé-sécurité au travail a été associée à un bien-être plus élevé des salariés dans la littérature, mais les mécanismes expliquant ce lien éventuel sont peu explorés. Notre cadre théorique néogramscien s'appuie sur la «politique de production» de Burawoy (1985). Nos analyses mobilisent une méthode mixte de recherche. A partir des enquêtes nationales SUMER 2003 et 2010, nous examinons l'influence d'une instance représentative du personnel en charge de la santé-sécurité, le Comité d'Hygiène, de Sécurité et de Conditions de Travail (CHSCT), sur le bien-être et sur les accidents du travail et testons l'effet médiateur de la mise en ouvre de politiques de prévention des risques professionnels. Toutes choses étant égales par ailleurs, la présence d'un CHSCT est associée de manière significative à une durée plus faible des absences pour accident du travail via la mise en œuvre de politique de prévention des risques professionnels. Toutefois, les résultats montrent que la présence d'un CHSCT est associée à une probabilité plus faible d'être satisfait au travail et à une fréquence plus élevée des accidents du travail. Une enquête qualitative par observation directe complète cette analyse en montrant l'importance du degré de mobilisation des acteurs membres du CHSCT et de leur activisme de la connaissance (Hall et alii, 2006). Nous discutons ces résultats et leurs implications managériales.

Mots clés : Instance représentative du personnel en santé-sécurité, Comité d'Hygiène, de Sécurité et de Conditions de Travail (CHSCT), santé au travail, bien-être au travail, prévention des risques professionnels, méthodes mixtes de recherche

\section{Remerciements :}

Nous remercions vivement nos évaluateurs anonymes pour leurs remarques et leurs suggestions qui ont permis d'améliorer substantiellement le contenu et la forme de notre article. 


\title{
The role of health and safety committees in influencing employees' well-being and work accidents. An exploratory study
}

\begin{abstract}
The presence of a health and safety committee in an organization was associated with higher employees' well-being in the literature, but the mechanisms underlying this possible link are little explored. Our theoretical framework is based on the "production politics" perspective of Burawoy (1985). Our analyses mobilize a mixed methods research design. From SUMER 2003 and 2010 national surveys, which respectively include 24486 employees and 47982 employees, we examine the effects of health and safety committees (CHSCT in French) on well-being and on work accidents and also test the mediating effect of the implementation of occupational risk prevention policies. All things being equal, the presence of a health and safety committee is significantly associated with lower length of absences for work accident via the implementation of an occupational risk prevention policy. However, the results demonstrate that the presence of a health and safety committee is associated with a lower likelihood of being satisfied by the job and a higher frequency of work accidents. A qualitative study trough a direct observation completes these analyses by showing the importance of the knowledge activism (Hall et al., 2006) of health and safety committees' members. We discuss these results and their managerial implications.
\end{abstract}

Key words: health and safety committee (CHSCT in French), health at work, well-being, occupational risk prevention policies, mixed methods design research. 


\section{Introduction}

La nouvelle loi relative au dialogue social et à l'emploi du 23 juillet 2015 (dite «loi de modernisation du dialogue social ») étend la possibilité d'instaurer une délégation unique du personnel aux entreprises de moins 300 salariés (au lieu de moins de 200 salariés auparavant) et permet aux entreprises de 300 salariés et plus de regrouper tout ou partie des IRP, institutions représentatives du personnel, par accords majoritaires. Certains voient là une remise en question du rôle du CHSCT (Comité d'Hygiène, de Sécurité et de Conditions de Travail) dans la vie des entreprises ${ }^{1}$. L'année 2012 fut marquée par la célébration de l'anniversaire des trente ans de la quatrième des lois dites «Auroux » instaurant les CHSCT. Leurs principales missions sont de contribuer à la protection de la santé physique et mentale et de la sécurité des travailleurs de l'établissement; de les informer sur les dangers auxquels ils peuvent être exposés comme sur les moyens de prévention ; de contribuer à l'amélioration des conditions de travail; de veiller à l'observation des prescriptions légales prises en ces matières (Brégier et Brégier, 2015). Pour mener à mener bien ces missions, les CHSCT disposent du droit d'enquêter pour analyser les risques auxquels sont exposés les salariés. L'ancien Ministre du Travail, Jean Auroux, tire le bilan suivant: «Je ne mesurais pas l'importance qu'auraient les CHSCT aujourd'hui. Je pensais qu'ils allaient fonctionner mais pas autant. Pour moi, c'est la traduction du durcissement des conditions de travail avec une compétition qui pèse sur les rythmes de travail, l'intensité du travail, des nouvelles technologies numériques qui exigent l’instantanéité (...) »(Auroux, 2012).

L'importance croissante du CHSCT constatée par Jean Auroux est également mise en évidence par les chercheurs. Ainsi, selon Moreau (2002, p. 825), «le CHSCT est en train de devenir un acteur essentiel sur lequel l'entreprise devra s'appuyer pour élaborer une véritable politique de santé ». Verkindt (2014), dans un rapport pour le Ministre du travail, de

\footnotetext{
${ }^{1}$ Source : «CHSCT : peut-on faire l'économie d'un lieu de débat sur le travail au sein de l'entreprise ? », Le Monde, 15 janvier 2015, collectif d'auteurs.
} 
l'emploi, de la formation professionnelle et du dialogue social, confirme l'importance prise par l'institution au cours de ces dernières années.

Les entreprises et établissements de 50 salariés et plus ont l'obligation de mettre en place un CHSCT selon l'article L. 4612-1 du Code du travail français. Pourtant, une partie seulement des établissements entrant dans le champ d'application de la loi s'y conforme et crée un CHSCT. Ainsi, seuls 81,1 \% des établissements employant de 50 à 499 salariés sont dotés d'un CHSCT, selon l'enquête SUMER 2010. A contrario, des entreprises et des établissements de moins de 50 salariés, non soumis à cette obligation, disposent d'un CHSCT. Respectivement $11,6 \%$ et $27 \%$ des établissements de moins de 9 salariés et de 10 à 49 salariés ont un CHSCT, selon l'enquête SUMER 2010. Ces pourcentages sont en progression, de 1,6 point et de 7 points, respectivement pour les établissements de moins de 9 salariés et de 10 à 49 salariés, comparativement aux résultats de l'enquête SUMER 2003.

L'objectif de cet article est de répondre à la question suivante : le CHSCT joue-t-il un rôle dans l'amélioration du bien-être des salariés et dans la réduction des accidents du travail au sein des entreprises ? L'étude de l'influence des IRP en charge de la santé-sécurité au travail sur le bien-être et sur les accidents du travail apparaît décisive dans une période marquée par une remise en question des prérogatives du CHSCT (Lerouge et Verkindt, 2015). Les recherches sur les liens entre organisation du travail et bien-être ont fait l'objet d'une vaste littérature (Luchman et Gonzales-Morales, 2013) mais rares sont les études analysant l'influence potentielle sur le bien-être des instances représentatives du personnel en charge de la santé-sécurité au travail. Le bien-être des salariés est un concept multidimensionnel (Biétry et Creusier, 2013 ; Ryan et Deci, 2001). A l'instar de Böckerman et alii (2012), Sparks et alii (2010), Tims et alii (2013), nous retiendrons la satisfaction au travail et la santé au travail comme dimensions du bien-être. Nous étudierons aussi l'influence de la présence d'un CHSCT sur les accidents du travail et testerons l'effet médiateur de la mise en œuvre de 
politiques de prévention des risques professionnels dans cette relation. La contribution théorique de cet article est d'améliorer la compréhension de l'influence du CHSCT sur le bien-être des salariés et les accidents du travail en nous appuyant sur la «politique de production » de Burawoy (1985). Notre recherche constitue une première étude exploratoire sur le sujet. Elle vise à proposer des implications managériales concernant le fonctionnement de cette instance mais aussi à mettre en évidence le rôle que peut jouer cette instance dans le co-développement de politiques de santé au travail.

Nous présentons tout d'abord notre modèle de recherche. Puis, nous exposons la méthodologie, les résultats de notre étude ainsi que leurs mises en relation avec ceux de l'analyse qualitative. Enfin, nous discutons les apports de la recherche et leurs implications managériales.

\section{L'influence des IRP en charge de la santé-sécurité au travail sur le bien-être des salariés et les accidents du travail : un modèle de recherche}

A l'instar de Coutrot (2009b) et de Hall et alii (2006), notre cadre théorique s'appuie sur la «politique de production» de Burawoy (1985). Dans ce cadre, nous étudions les IRP en charge de la santé-sécurité au travail comme des lieux d'affrontements politiques et idéologiques engageant les directions d'entreprise et les représentants des salariés dans des stratégies politiques de domination. Selon Hall et alii (2006), deux déterminants apparaissent cruciaux dans la production d'effets positifs sur la santé et la sécurité des salariés par les IRP en charge de la santé-sécurité au travail : des mécanismes d'ordre cognitif (effet connaissance ou «Worker knowledge»), et d'ordre politique (effet pouvoir ou «Worker power»). Les mécanismes d'ordre cognitif posent l'idée que les salariés et leurs représentants peuvent agir sur les questions de santé et de sécurité au travail s'ils ont la capacité d'identifier et de comprendre les risques professionnels. Les mécanismes d'ordre politique renvoient à la question du pouvoir des salariés et de leurs représentants face aux directions. Ces mécanismes 
incluent de nombreux aspects, que Freeman et Medoff (1984) qualifieraient, dans leur théorie du comportement syndical, de collective voice du syndicalisme : la possibilité de contrer l'idéologie dominante, le degré d'union des salariés et des syndicats, la possibilité de faire respecter le cadre légal et les protections des salariés, au sein d'une entreprise (Hall et alii 2006). Comme le note Coutrot (2009b, p. 26), «l'implication des représentants du personnel dans le domaine de la "santé-sécurité » peut contribuer à focaliser l'action représentative sur les questions de santé-sécurité, renforçant la pression sur le management pour l'adoption de politiques de prévention efficaces ». Nous présentons, dans les paragraphes suivants, les hypothèses de notre modèle de recherche.

\section{1 Les relations entre la présence d'une IRP en charge de la santé-sécurité au travail et la satisfaction au travail}

Les IRP en charge de la santé-sécurité agissent, par des mécanismes d'ordre cognitif (effet connaissance) et d'ordre politique (effet pouvoir), sur la satisfaction au travail. Les IRP en charge de la santé-sécurité, en contribuant à améliorer la connaissance par les salariés des risques professionnels auxquels ils sont exposés (effet connaissance), sont susceptibles, dans un second temps, d'inciter les salariés à faire pression (effet pouvoir), via leurs représentants, sur la direction afin d'améliorer leurs conditions de travail. Or, l'amélioration des conditions de travail a des effets positifs sur la satisfaction au travail (Böckerman et alii, 2009 ; Bouville 2009). Toutefois, rares sont les études en sciences de gestion, en psychologie, en sociologie du travail ou en épidémiologie ${ }^{2}$ portant sur les liens entre la présence d'une instance représentative du personnel en matière de santé-sécurité et la satisfaction au travail. A notre connaissance, seules deux études ont analysé cette relation (Torp et Moen, 2006 ; Relihan et alii, 2009). La recherche norvégienne de Torp et Moen (2006) montre que « la satisfaction

\footnotetext{
${ }^{2}$ Pour effectuer notre recherche documentaire sur ce domaine, nous avons utilisé les bases de données Business Source Complete (Ebsco), Blackwell Synergy, British Medical Journal Group, Elsevier ScienceDirect, Medline (PubMed), Psycarticles, PsycInfo, Psychology and Behavorial Sciences Collection, Springer Link, Wiley Interscience, Google Scholar avec les combinaisons des mots clés suivants : " safety representative », "worker representation », «job satisfaction ».
} 
des salariés vis-à-vis de leur environnement psychosocial », variable qui inclut trois dimensions (la coopération, le stress et la satisfaction au travail), est corrélée positivement au renforcement des activités de l'instance en charge de la santé et de l'hygiène au travail. L'étude de Relihan et alii (2009) montre que lorsque la politique de prévention et de sécurité des risques professionnels (dénommée safety climate dans leur recherche) est ressentie par les salariés comme fortement développée, les scores de satisfaction au travail déclarés par les salariés sont élevés. Or, la présence de CHSCT contribue fortement à la qualité des politiques de prévention des risques professionnels (Coutrot, 2009b). Nous posons donc l'hypothèse suivante :

H1: La présence d'une instance représentative du personnel en matière de santé-sécurité est associée positivement à la satisfaction au travail.

\subsection{Les relations entre la présence d'une IRP en charge de la santé-sécurité au travail et la santé au travail}

Les IRP en charge de la santé-sécurité agissent sur la santé au travail des salariés, également par des mécanismes d'ordres cognitif et politique. Ces IRP contribuent à renforcer la prise de conscience par les représentants des salariés des risques auxquels ceux-ci sont exposés dans le cadre de leur travail (effet connaissance). Forts de cette connaissance, les représentants, en mobilisant les salariés, l'inspection du travail ou des experts en risques professionnels, exercent une pression politique (effet pouvoir) sur la direction afin qu'elle mette en place des politiques visant à diminuer l'exposition des salariés aux risques professionnels, améliorant in fine la santé des salariés. Selon Walters et Frick (2000), l'effet positif de la présence d'une IRP en charge de la santé-sécurité au travail sur la santé au travail est reconnu dans la littérature. Sur la base de nombreuses études de cas dans plusieurs pays, Walters et Nichols (2009) confirment cette relation positive. Nous faisons donc l'hypothèse suivante : 
$H 2$ : La présence d'une instance représentative du personnel en matière de santé-sécurité est associée positivement à la santé au travail.

\subsection{Les relations entre la présence d'une IRP en charge de la santé-sécurité au travail et les accidents du travail}

Les IRP en charge de la santé-sécurité agissent théoriquement de deux manières sur les accidents du travail : 1. en renforçant le rapport de force en faveur des salariés et de leurs représentants (effet pouvoir), ils limitent les risques de sous-déclaration des accidents du travail, phénomène observé par plusieurs études internationales (Amossé et alii, 2012; Walters et Nichols (2007); 2. en faisant prendre conscience par les salariés des risques professionnels auxquels ils sont exposés (effet connaissance), ce qui les conduit, ainsi que leurs représentants, à revendiquer et à obtenir des politiques de prévention de risques professionnels, qui limitent la gravité des accidents du travail et ainsi leur durée. Il s'agit d'un champ de recherche en développement. Peu d'auteurs s'y sont intéressés (Nichols et alii, 2007; Popma, 2009). Les études de Nichols et alii (2007), à partir de l'enquête nationale anglaise WIRS-WERS90 ${ }^{3}$, et de Popma (2009), concluent que la présence d'une IRP en charge de la santé-sécurité au travail contribue à diminuer la durée des absences pour accident du travail. Ces résultats pourraient s'expliquer par le déploiement plus systématique de politiques de prévention des problèmes de santé au travail dans les entreprises dotées d'une IRP en charge de la santé-sécurité au travail (Popma, 2009). De même, Coutrot (2009a) postule que l'action du CHSCT contraint la direction à renforcer les politiques de prévention des risques professionnels auxquels sont exposés les salariés. Toutefois, ces rares études comportent une limite importante. Elles ne contrôlent pas l'influence des effets des conditions de travail et des caractéristiques structurelles (secteur d'activité, niveau de qualification des salariés...). En effet, les conditions de travail et les variables «structurelles» (taille de

\footnotetext{
${ }^{3}$ L'équivalent britannique de l'enquête française REPONSE.
} 
l'établissement, secteur d'activité...) sont des déterminants de l'implantation des instances représentatives du personnel en matière de santé au travail (Coutrot, 2009a, 2009b). Coutrot (2009b, p. 32) a ainsi montré que «les directions tendent d'autant plus à mettre en place un CHSCT que les risques professionnels sont importants dans leur établissement, du fait des caractéristiques techniques et organisationnelles de leur établissement ». De même, la taille de l'établissement ainsi que la nature du secteur d'activité influencent la décision d'implantation d'un CHSCT (Coutrot, 2009a, 2009b). Par conséquent, ces variables peuvent exercer un effet de confusion sur les relations testées.

Nous supposons donc que l'effet pouvoir des IRP en charge de la santé-sécurité diminue la sous-déclaration des accidents du travail et, ainsi, influence positivement la fréquence des absences pour accident du travail. Par ailleurs, l'effet connaissance contribue à améliorer les politiques de prévention des risques professionnels et participe ainsi à la diminution de la durée des absences pour accident du travail. Nous posons donc les hypothèses suivantes :

$H 3 a$ : La présence d'une instance représentative du personnel en matière de santé-sécurité est associée positivement à la fréquence des absences pour accident du travail.

$H 3 b$ : La présence d'une instance représentative du personnel en matière de santé-sécurité est associée négativement à la durée des absences pour accident du travail.

$H 3 c:$ La présence d'une instance représentative du personnel en matière de santé-sécurité est associée négativement à la durée des accidents du travail par l'intermédiaire de politiques de prévention des risques professionnels.

\subsection{Le modèle de recherche}

L'ensemble de ces hypothèses sont résumés dans notre modèle de recherche. Ce modèle examine les relations, entre la présence d'une IRP en charge de la santé-sécurité au travail et 
le bien-être des salariés, entre la présence de cette IRP et la fréquence ou la durée des accidents du travail ainsi que la médiation de cette relation (figure 1).

Figure 1. Modèle de recherche

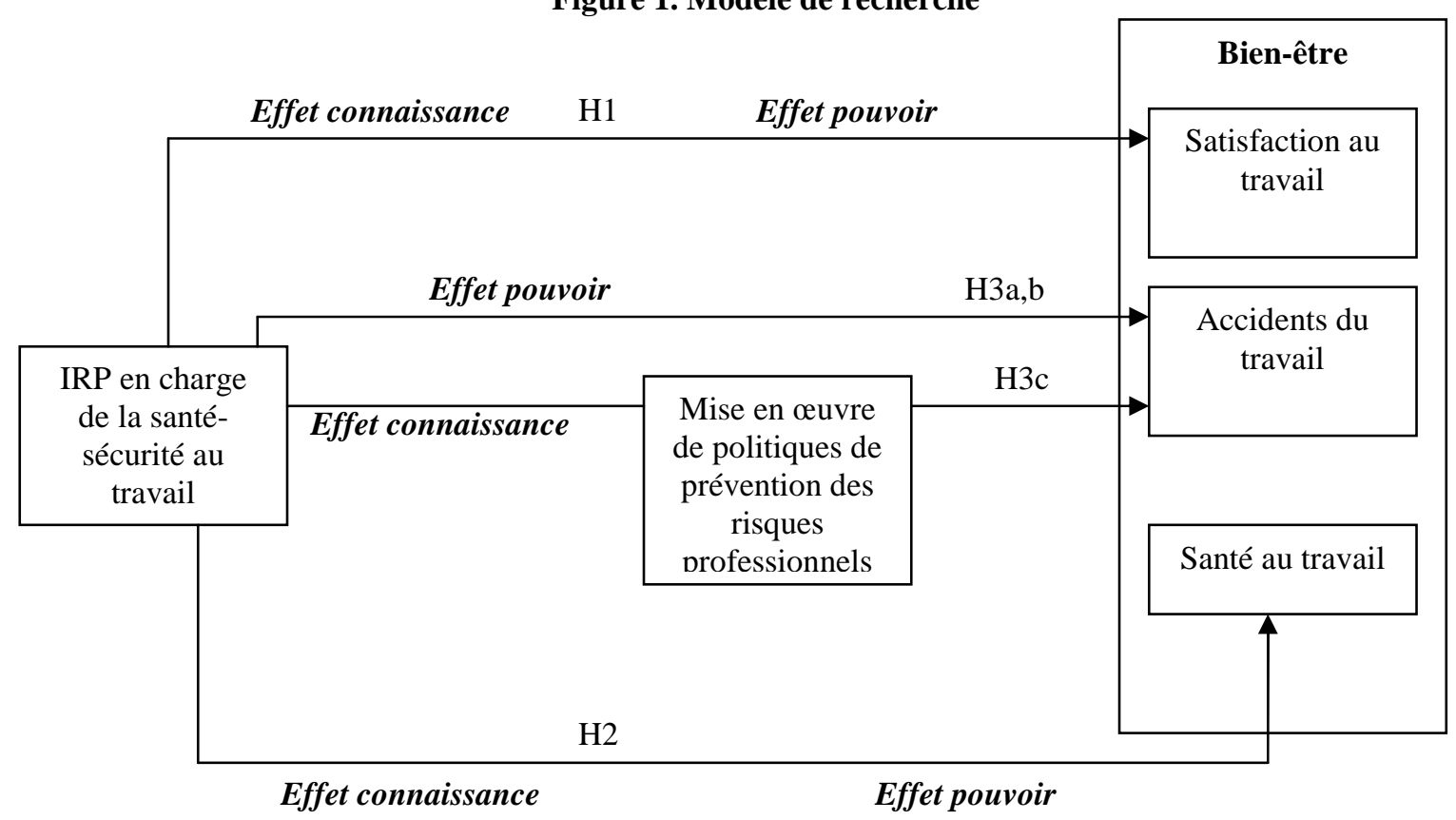

\section{Méthodologie : une méthode mixte de recherche}

Nous avons mobilisé une méthodologie de recherche mixte et, plus spécifiquement, un protocole (ou design) explicatif (Creswell et Plano Clark, 2011). Ce protocole renvoie à une conception séquentielle de la méthodologie mixte dans laquelle les résultats quantitatifs sont expliqués à l'aide des données qualitatives. Cette démarche a pour objectif d'éclairer et d'expliquer plus précisément les résultats liés à l'hypothèse $H 3 c$. Nous présentons successivement: les enquêtes SUMER 2003 et 2010 sur lesquelles repose notre analyse quantitative, les mesures des variables explicatives, des variables à expliquer et des variables de contrôle, la méthodologie de notre analyse statistique ainsi que de notre observation directe. 


\subsection{Présentation des enquêtes SUMER 2003 et SUMER 2010 et des mesures des variables}

Nous avons eu accès aux données des enquêtes SUMER 2003 et 2010, dans le cadre d'un projet de recherche plus vaste, formalisé par une convention passée, après examen, entre notre laboratoire de recherche et le Centre Maurice Halbwachs - Archives de Données Issues de la Statistique Publique. L'enquête SUMER 2003 a été réalisée de juin 2002 à décembre 2003. 1792 médecins du travail, représentant plus de $20 \%$ des médecins du travail français, ont choisi au hasard 56314 salariés dont 49984 ont répondu au questionnaire. Le champ d'application de l'enquête comprend l'ensemble des salariés surveillés par la médecine du travail du régime général et de la Mutualité Sociale Agricole. Elle inclut aussi les salariés des hôpitaux publics, d'EDF-GDF (Electricité et Gaz de France), de La Poste, de la SNCF et d'Air France. Son champ d'application ne couvre toutefois pas les fonctions publiques territoriales et d'Etat, le secteur des transports, des mines, de la pêche et des télécommunications (France Télécom). L'enquête couvre 17,5 millions des 21,7 millions des salariés travaillant en France (soit $80 \%$ des salariés). L'enquête SUMER 2010 est la troisième enquête SUMER. Elle a eu lieu avec la collaboration de 2400 médecins du travail enquêteurs. La collecte a été réalisée de janvier 2009 à la mi-2010. Comme SUMER 2003, elle couvre le champ des salariés suivis par la médecine du travail et couverts par le régime général et le régime agricole (MSA), ainsi que les salariés d'Air France, de la SNCF, des groupes EDF et GDF Suez, de La Poste, des grandes régies de transport urbain et des Hôpitaux publics. SUMER 2010 élargit également son champ à la Fonction publique d'Etat, à titre expérimental, à la fonction territoriale, aux gens de mer ainsi qu'aux salariés de la Réunion. L'enquête Sumer 2010 est représentative de près de 22 millions de salariés, soit 92 $\%$ des salariés. Les tailles de nos deux échantillons sont respectivement, pour les enquêtes SUMER 2003 et 2010, de 24486 salariés et de 47982 salariés. Les deux enquêtes SUMER 2003 et 2010 ont pour point commun d'être représentatives de la population salariée 
française. Enfin, signalons que certaines questions portant sur la santé au travail, présentes dans l'enquête SUMER 2003 (voir les indicateurs 2, 3 et 4 ci-dessous) n'ont pas été reprises dans l'enquête SUMER 2010.

\subsubsection{Mesures de la variable explicative et des variables dépendantes}

La présence ou l'absence d'un CHSCT (dans les enquêtes SUMER 2003 et 2010) a été mesurée au travers de l'item suivant: «Existe-t-il un comité d'hygiène, de sécurité et des conditions de travail (CHSCT) dans vos établissements?». Les réponses possibles étaient «oui » ou «non».

La satisfaction au travail (dans les enquêtes SUMER 2003 et 2010) a été mesurée par l'item «Dans l'ensemble, je suis satisfait de mon travail ». Les participants répondaient sur une échelle à 4 points. Ce choix se justifie car une échelle de satisfaction globale au travail à un item ne se traduit pas par une diminution importante de la fiabilité comparativement à une échelle à plusieurs items (Warnous et alii, 1997).

La santé au travail (dans l'enquête SUMER 2003 pour l'ensemble des indicateurs et, dans l'enquête SUMER 2010, pour l'indicateur 1) a été mesurée par quatre indicateurs. Un premier indicateur concerne les perceptions globales des salariés sur les atteintes à la santé considérées comme dues au travail («Mon travail est plutôt mauvais pour ma santé »).

Une seconde catégorie d'indicateurs porte sur différents types d'atteintes à la santé au travail que déclarent les salariés. Un item porte sur une affection physique : la fatigue. Dans l'acception courante, le terme «fatigant » renvoie en effet au travail manuel physiquement épuisant (Bué et alii, 2008). Deux items portent sur des affections d'ordre psychologique : le stress, l'insomnie.

(Indicateur 1) «Pensez-vous que votre travail est mauvais pour votre santé?». Les participants devaient choisir une réponse parmi les trois suivantes : 1. «Non, mon travail 
n’influence pas ma santé » (codée 1) ; 2. «Oui, mon travail est plutôt bon pour ma santé » (codée 2) ; 3. «Oui, mon travail est plutôt mauvais pour ma santé » (codée 3). Nous avons traité cette variable comme une variable ordinale dans la suite de notre analyse.

(Indicateur 2) «Il m'arrive de ne pas dormir car je pense à mon travail». Les participants répondaient sur une échelle à 4 points.

(Indicateur 3) «Dans l'ensemble, estimez-vous que votre travail est fatigant?».

(Indicateur 4) «Dans l'ensemble, estimez-vous que votre travail est stressant?».

Les participants ont répondu sur une échelle à 10 points pour les deux dernières questions. Les indicateurs subjectifs de l'état de santé sont fiables par comparaison à des mesures objectives de l'état de santé (Kaplan et alii, 1996 ; Singh-Manoux et alii, 2006).

Les accidents du travail (dans l'enquête SUMER 2010) ont été mesurés par deux indicateurs: la durée des absences pour accident du travail $(m=6,5 ; \quad \sigma=29,5)$ et la fréquence des accidents du travail qui est une variable ordinale composée de quatre modalités : «pas d'accident » (codée 0) ; «1 accident » (codée 1) ; «2 accidents » (codée 2) ; «3 accidents ou plus» (codée 3). Notre indicateur est construit sur la base d'autodéclarations. Cependant des recherches ont montré que le niveau de convergence des mesures des absences par l'auto-déclaration et des mesures basées sur un recensement des absences par l'intermédiaire d'une base de données approchait $70 \%$ (Ferrie et alii, 2005 ; Voss et alii, 2008).

La politique de prévention des risques professionnels (dans l'enquête SUMER 2010) a été mesurée par la question suivante: «Au cours des 12 derniers mois, y-a-t'il eu une intervention d'IPRP (Intervenants en Prévention des Risques Professionnels) (appartenant ou non au Service de Santé au Travail) ou d'autres consultants dans l'établissement ?». Les 
médecins du travail interrogés dans le cadre de l'enquête SUMER 2010 devaient répondre par «oui » ou par «non ».

\subsubsection{Les variables de contrôle}

Nous avons introduit des variables de contrôle afin d'éviter les effets de confusion. Ces derniers consistent à attribuer la cause d'un effet à la variable explicative testée (dans notre cas, la présence d'un CHSCT) alors que cet effet est expliqué par une autre ou d'autres variables (Mbengue et Vandangeon-Derumez, 2003). Nous avons donc contrôlé les effets d'un ensemble de variables qui peuvent être des déterminants de l'implantation d'un CHSCT tout en ayant des effets sur nos variables dépendantes (voir annexe).

\subsubsection{Analyse statistique}

Nous avons effectué des régressions logistiques ordinales lorsque la variable dépendante était de type ordinal, ce qui était le cas de la satisfaction au travail, de la santé au travail et de la fréquence des accidents du travail. Pour tester l'effet de la présence d'un CHSCT sur la mise en œuvre d'une politique de prévention des risques professionnels, nous avons utilisé une régression logistique binomiale. Enfin, compte tenu de la nature des données pour la durée des absences pour accident du travail (données de comptage d'événements relativement rares), nous avons opté pour le modèle de régression suivant la loi binomiale négative car il présentait un meilleur ajustement à nos données que le modèle de régression suivant la loi de Poisson. Afin de nous prémunir contre les problèmes de multicollinéarité, nous nous sommes assuré que les variables explicatives n'étaient pas colinéaires, ceci afin d'éviter que les variables explicatives aient une corrélation supérieure à 0,7 en valeur absolue (Tenenhaus, 2007). Pour tester l'effet de la variable médiatrice, nous avons utilisé la procédure de Baron et Kenny (1986). Nous n'avons pas mobilisé la procédure de test de médiation développée, plus récemment, par Preacher et Hayes (2004) car elle repose sur les hypothèses d'une variable 
dépendante d'intervalle et l'utilisation d'un modèle de régression reposant sur l'estimation par les moindres carrés ordinaires. Or, dans notre cas, la variable dépendante est une variable de comptage et le modèle de régression est binomial négatif. Par ailleurs, nous avons testé s'il existait des problèmes d'endogénéité dans nos modèles en suivant la méthodologie de Foster (1997). Nous avons donc vérifié que la variable explicative de notre modèle, Présence d'un CHSCT, n'était corrélée avec le terme d'erreur $\varepsilon$ dans aucune de nos régressions ayant pour variables à expliquer, la satisfaction au travail ou la durée/fréquence des accidents du travail.

\subsection{Analyse qualitative par observation directe}

Nous avons complété notre analyse quantitative par une enquête qualitative par observation non participante.

Nous avons réalisé deux demi-journées d'observation directe à découvert, à temps plein, le 20 et le 30 janvier 2013, lors de deux réunions de CHSCT de deux établissements d'une même entreprise de collecte d'ordures ménagères, composés respectivement de 289 éboueurs pour le premier établissement (CHSCT 1), et de 295 éboueurs pour le second (CHSCT 2). Etaient présents lors de ces deux réunions : les directeurs d'établissement, assumant la présidence, une délégation du personnel (3 à 5 salariés de l'entreprise par CHSCT), les membres avec voix consultatives (un représentant du service prévention de la Caisse Régionale d'Assurance Maladie $^{4}$, deux préventeurs de l'entreprise). Ce nombre de jours d'observation résulte d'une négociation avec la direction. Nous avons suivi une démarche méthodologique rigoureuse (Wacheux, 1996 ; Arborio et Fournier, 1999). Nous avons rédigé des notes repères (constituées de propos échangées, de postures et attitudes adoptées par les différents acteurs, des non-dits...), un journal de terrain, ainsi qu'un journal d'après journal (Arborio et Fournier, 1999) et croisé nos analyses avec celles d'un autre chercheur ayant assisté aux deux

\footnotetext{
${ }^{4}$ Les Caisses d'assurance retraite et de la santé au travail (Carsat) sont entrées en vigueur à compter du 1er juillet 2010. Elles remplacent les anciennes Caisses régionales d'assurance maladie (Cram), à l'exception de la région Ile-de-France.
} 
réunions. Le journal de terrain contient des descriptions de ce que nous avons vu ou entendu, ainsi que le recueil du « lexique indigène » (Arborio et Fournier, 1999), des conversations et des témoignages. Lors de l'observation, nous avons adopté une attitude d'ouverture, de disponibilité et exercé notre faculté d'étonnement (Arborio et Fournier, 1999).

L'objectif de cette observation directe était, tout d'abord, d'éclairer, par une approche qualitative, les régularités statistiques observées à partir des données des enquêtes SUMER 2003 et 2010, ensuite, de mieux comprendre en quoi les caractéristiques des acteurs présents lors d'une réunion de CHSCT, leurs interactions, peuvent influencer les relations entre la présence d'un CHSCT et la durée des accidents du travail. L'observation directe du fonctionnement de deux CHSCT, au sein de deux établissements d'une même entreprise, permettait de comparer la manière dont l'activisme des acteurs en présence peut jouer sur la plus ou moins grande efficacité d'un CHSCT.

\section{Résultats}

Nous présentons les résultats obtenus quant aux liens entre, d'une part, la présence d'un CHSCT et, d'autre part, la satisfaction au travail, la santé au travail et les absences pour accident du travail. Une analyse descriptive de la présence d'un CHSCT par taille d'établissement et par secteur d'activité montre quelques évolutions sensibles entre les enquêtes SUMER 2003 et 2010. La part des établissements dont l'effectif est compris entre 10 et 49 salariés (soit en-dessous du seuil légal de 50 salariés et plus, au-delà duquel la présence d'un CHSCT est obligatoire), et dans lesquels sont présents un CHSCT, augmente de $20,4 \%$ à $26,9 \%$ entre 2003 et 2010 . Une évolution identique est observée dans le secteur tertiaire : la part des établissements du secteur tertiaire, dans lesquels est présent un CHSCT, augmente de 55,2\% à 62,2\% entre 2003 et 2010. 


\subsection{Les liens entre la présence d'un CHSCT et la satisfaction ou la santé au travail}

Dans les tableaux $1 \mathrm{a}$ et $1 \mathrm{~b}$, nous avons présenté les résultats des régressions logistiques ordinales.

De manière inattendue, l'hypothèse 1 n'est pas vérifiée, la présence d'un CHSCT est associée à une probabilité plus faible d'être satisfait au travail $(\beta=-0,368, p<0,01$, pour l'enquête SUMER 2003; tableau 1a ; $\beta=-0,296, p<0,01$, pour l'enquête SUMER 2010 ; tableau 1a).

L'hypothèse 2 est partiellement validée : l'effet de la présence d'un CHSCT sur la santé au travail varie en fonction de l'indicateur de mesure de la santé au travail. La présence d'un CHSCT conduit à réduire le risque pour un salarié de ne pas dormir à cause de son travail $(\beta=$ - 0,072, $p<0,05$, pour l'enquête SUMER 2003 ; tableau 1b).

Tableau 1a. Les effets de la présence d'un CHSCT sur la satisfaction au travail : résultats de régressions logistiques ordinales (Paramètres estimés et test de Wald)

\begin{tabular}{|l|c|c|}
\hline Variables dépendantes & $\beta$ & Satisfaction au travail \\
\hline & $-0,368$ & 0,000 \\
Présence d'un CHSCT & $* * *$ & \\
Enquête SUMER 2003 & & 0,000 \\
\hline Présence d'un CHSCT Enquête & $-0,296$ & \\
SUMER 2010 & $* * *$ & \\
\hline
\end{tabular}

$* * * \mathrm{p}<, 01 * * \mathrm{p}<, 05 * \mathrm{p}<, 1 \mathrm{~ns}:$ paramètre estimé non significatif. $p$ représente la valeur-p. UMER 2010.

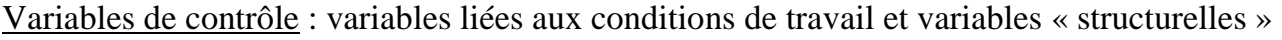


Tableau 1b. Les effets de la présence d'un CHSCT sur les différents indicateurs de mesure de la santé au travail: résultats de régressions logistiques ordinales (Paramètres estimés et test de Wald)

\begin{tabular}{|c|c|c|c|c|c|c|c|c|}
\hline Variables & \multicolumn{8}{|c|}{ Indicateurs de mesure de la santé au travail } \\
\hline \multirow[b]{2}{*}{$\begin{array}{l}\text { Présence } \\
\text { d'un } \\
\text { CHSCT } \\
\text { Enquête } \\
\text { SUMER } \\
2003\end{array}$} & \multicolumn{2}{|c|}{$\begin{array}{l}\text { Indicateur } 1 \text { : déclarer } \\
\text { un lien subjectif entre } \\
\text { travail et mauvaise } \\
\text { santé }\end{array}$} & \multicolumn{2}{|c|}{$\begin{array}{l}\text { Indicateur } 2 \text { : déclarer } \\
\text { ne pas dormir à cause } \\
\text { du travail }\end{array}$} & \multicolumn{2}{|c|}{$\begin{array}{l}\text { Indicateur } 3 \text { : travail } \\
\text { déclaré fatigant }\end{array}$} & \multicolumn{2}{|c|}{$\begin{array}{l}\text { Indicateur } 4 \text { : travail } \\
\text { déclaré stressant }\end{array}$} \\
\hline & $\begin{array}{c}0,067 \\
*\end{array}$ & $\begin{array}{c}p \\
0,059\end{array}$ & $\begin{array}{c}\beta \\
\\
-0.072 \\
* *\end{array}$ & $\begin{array}{c}p \\
0,032\end{array}$ & $\begin{array}{c}\beta \\
0,024 \\
(\mathrm{~ns})\end{array}$ & $\begin{array}{c}p \\
0,448\end{array}$ & $\begin{array}{c}\beta \\
0,103 \\
* * *\end{array}$ & $\begin{array}{c}p \\
0,001\end{array}$ \\
\hline $\begin{array}{l}\text { Présence } \\
\text { d'un } \\
\text { CHSCT } \\
\text { Enquête } \\
\text { SUMER } \\
2010\end{array}$ & $\begin{array}{l}0,130 \\
* * *\end{array}$ & 0,000 & -1 & & -1 & & -1 & \\
\hline
\end{tabular}

Toutefois, l'effet sur le travail fatigant n'est pas significatif $(\beta=0,024, p>0,1$, pour l'enquête SUMER 2003 ; tableau 1b). La présence d'un CHSCT contribue à faire ressentir le travail comme plus stressant $(\beta=0,103, p<0,01$, pour l'enquête SUMER 2003 ; tableau 1b) et à percevoir le travail comme mauvais pour la santé $(\beta=0,067, p<0,1$, pour l'enquête SUMER 2003; tableau $1 \mathrm{~b} ; \beta=0,130, p<0,01$, pour l'enquête SUMER 2010 ; tableau 1b). Pour ce dernier indicateur, la présence d'un CHSCT a un effet important sur la probabilité de percevoir le travail comme mauvais pour la santé. Notons que l'importance de l'effet de la présence d'un CHSCT sur la probabilité de percevoir le travail comme mauvais pour la santé a considérablement augmenté entre les deux vagues d'enquêtes SUMER 2003 et 2010.

\subsection{Les liens entre la présence d'un CHSCT et les accidents du travail}

Dans le tableau 2, nous avons présenté les résultats du modèle de régression binomial négatif. Les hypothèses $3 a$ et $3 b$ sont confirmées. La présence d'un CSHCT est associée significativement à une fréquence plus élevée des accidents du travail déclarés $(\beta=0,226, p<$ 
0,01, modèle 2, tableau 2). L'hypothèse 3a est validée. La présence d'un CSHCT est associée significativement une durée plus faible des absences pour accident du travail $(\beta=-0,070, p<$ 0,05, modèle 1 , tableau 2). L'hypothèse $3 b$ est confirmée.

Tableau 2. Les effets de la présence d'un CHSCT sur les accidents du travail: par des modèles de régression binomiaux négatifs pour les modèles 1 et 3 , par une régression logistique ordinale pour le modèle 2 (Paramètres estimés et test de Wald)

\begin{tabular}{|c|c|c|c|c|c|c|}
\hline Variables & \multicolumn{6}{|c|}{ Accidents du travail (AT) } \\
\hline \multirow[b]{2}{*}{$\begin{array}{l}\text { Présence } \\
\text { d'un } \\
\text { CHSCT } \\
\text { Enquête } \\
\text { SUMER } \\
2010\end{array}$} & \multicolumn{2}{|c|}{$\begin{array}{c}\text { Modèle 1 } \\
\text { (durée des AT) }\end{array}$} & \multicolumn{2}{|c|}{$\begin{array}{c}\text { Modèle 2 } \\
\text { (fréquence des AT) }\end{array}$} & \multicolumn{2}{|c|}{$\begin{array}{c}\text { Modèle } 3 \\
\text { (durée des AT via } \\
\text { médiateur) }\end{array}$} \\
\hline & $\begin{array}{c}-0,070 \\
* *\end{array}$ & $\begin{array}{c}p \\
0,028\end{array}$ & $\begin{array}{c}0,226 \\
* * *\end{array}$ & 0,000 & $\begin{array}{c}-0,014 \\
(\mathrm{~ns})\end{array}$ & 0,714 \\
\hline
\end{tabular}

$* * * \mathrm{p}<, 01 * * \mathrm{p}<, 05 * \mathrm{p}<, 1$ ns : paramètre estimé non significatif. $p$ représente la valeur-p. Variables de contrôle : variables liées aux conditions de travail et variables «structurelles »

Tableau 3. Les effets de la présence d'un CHSCT sur la mise en œuvre de politiques de prévention (médiateur) : régression logistique binomiale (Paramètres estimés et test de Wald)

\begin{tabular}{|l|c|c|}
\hline Variables dépendantes & \multicolumn{2}{|c|}{ Existence d'une politique de prévention des risques professionnels } \\
\hline Présence d'un CHSCT & $\beta$ & 0,000 \\
Enquête SUMER 2010 & 2,220 & \\
\hline
\end{tabular}

$* * * \mathrm{p}<, 01 * * \mathrm{p}<, 05 * \mathrm{p}<, 1$ ns : paramètre estimé non significatif. $p$ représente la valeur-p. Variables de contrôle : variables liées aux conditions de travail et variables «structurelles »

Selon la première étape de la procédure de test d'un effet médiateur de Baron et Kenny (1986), nous avons testé la relation entre la présence d'un CHSCT et la mise en œuvre d'une politique de prévention des risques professionnels (tableau 3). La présence d'un CHSCT a un effet significatif très important sur la probabilité d'une intervention d'IPRP (Intervenants en Prévention des Risques Professionnels) (appartenant ou non au Service de Santé au Travail) ou d'autres consultants dans l'établissement. La deuxième étape correspond à la régression de la variable dépendante sur la variable indépendante (voir le résultat présenté dans le modèle 1 
du tableau 2). Selon la dernière étape de la procédure de test d'un effet médiateur de Baron et Kenny (1986), nous avons analysé l'évolution du paramètre estimé, associé à la variable «présence d'un CHSCT», lorsque nous introduisons dans la régression la variable médiatrice « politique de prévention » (modèle 3, tableau 2). La valeur du paramètre estimé, associé à la variable «Présence d'un CHSCT», diminue en présence du médiateur «politique de prévention» pour la durée des absences pour accident du travail $(\beta=-0,070, p<0,05$, modèle $1->\beta=-0,014, p>0,1 ;$ modèle 3, tableau 2). L'hypothèse $3 c$ est confirmée. Le tableau 4 récapitulatif présente les hypothèses validées ou infirmées.

Tableau 4. Synthèse des hypothèses validées ou infirmées

\begin{tabular}{|l|c|}
\hline \multicolumn{1}{|c|}{ Hypothèses } & Hypothèses validées ou infirmées \\
\hline $\begin{array}{l}\text { H1 : La présence d'une instance représentative du personnel en } \\
\text { matière de santé-sécurité est associée positivement à la } \\
\text { satisfaction au travail. }\end{array}$ & H1 infirmée \\
\hline $\begin{array}{l}\text { H2: La présence d'une instance représentative du personnel en } \\
\text { matière de santé-sécurité est associée positivement à la santé } \\
\text { au travail. }\end{array}$ & H2 partiellement validée \\
\hline $\begin{array}{l}\text { H3a : La présence d'une instance représentative du personnel } \\
\text { en matière de santé-sécurité est associée positivement à la } \\
\text { fréquence des absences pour accident du travail. }\end{array}$ & H3a validée \\
\hline $\begin{array}{l}\text { H3b : La présence d'une instance représentative du personnel } \\
\text { en matière de santé-sécurité est associée négativement à la } \\
\text { durée des absences pour accident du travail }\end{array}$ & H3b validée \\
\hline $\begin{array}{l}\text { H3c: La présence d'une instance représentative du personnel } \\
\text { en matière de santé-sécurité est associée négativement à la } \\
\text { durée des accidents du travail via les politiques de prévention } \\
\text { des risques professionnels. }\end{array}$ & H3c validée \\
\hline
\end{tabular}

\section{Approfondissement et mise en perspective des résultats quantitatifs à l'aune de ceux de l'analyse qualitative}

Nous approfondissons et éclairons les résultats issus de notre analyse quantitative à la lumière de ceux de notre observation directe.

\subsection{L'effet contre-intuitif de la présence d'un CHSCT sur la satisfaction au travail : quelles explications?}

La présence d'un CHSCT dans un établissement susciterait de telles attentes chez les salariés qu'elles expliqueraient le résultat paradoxal d'un lien négatif entre la présence d'un CHSCT 
et la satisfaction au travail. Le CHSCT contribuerait à alerter les salariés sur les effets négatifs de leur travail sur leur santé (rappelons que la présence d'un CHSCT contribue à faire percevoir par les salariés le travail comme mauvais pour la santé : $\beta=0,067, p<0,5$, pour l'enquête SUMER 2003; tableau 3b; $\beta=0,130, p<0,01$, pour l'enquête SUMER 2010 ; tableau 3b) mais serait impuissant à y remédier. L'effet connaissance (cf. figure 1) susciterait des attentes fortes auprès des salariés mais cet effet, n'étant pas suivi d'une amélioration des conditions de travail par impuissance politique des représentants des salariés (c'est-à-dire que l'effet pouvoir serait faible, voire inexistant), contribuerait à une insatisfaction au travail des salariés. Ce dernier résultat vaut quelque soit la catégorie socio-professionnelle du salarié, ce qui conforte notre invalidation de l'hypothèse 1. En effet, des analyses de régression logistique ordinale sur la satisfaction au travail, menées à partir des données de l'enquête SUMER 2010, sur différentes catégories socio-professionnelles (cadres, ouvriers, employés), aboutissent systématiquement à des liens significatifs et négatifs entre la présence d'un CHSCT et la satisfaction au travail.

\subsection{Les effets bénéfiques de la présence d'un CHSCT sur la santé au travail : oui, mais par quel canal?}

La présence d'un CHSCT conduit à réduire le risque pour un salarié de ne pas dormir à cause de son travail. Ce lien significatif entre la présence d'un CHSCT et une meilleure santé au travail est congruent avec les conclusions de Walters et Frick (2000). Toutefois, de manière inattendue, cette relation ne joue, dans le sens attendu, que pour un des indicateurs de mesure de la santé au travail parmi les quatre utilisés dans notre recherche. L'effet bénéfique du CHSCT sur la santé au travail s'exercerait par le canal de l'information (Coutrot, 2009b). En effet, le CHSCT joue un rôle important d'information auprès des salariés au sujet de leurs droits tel que, par exemple, le droit de retrait (droit pour un salarié confronté à un danger imminent pour sa vie ou pour sa santé de cesser son activité et, si nécessaire, de quitter les 
lieux pour se mettre en sécurité). Les salariés déclarent plus souvent recevoir une information sur les risques au cours des douze derniers mois dans les établissements possédant un CHSCT que dans ceux qui en sont dépourvus $(25 \%$ contre $12 \%$ pour les établissements sans CHSCT $)^{5}$. Enfin, la médiation entre la présence d'un CHSCT et la santé au travail pourrait s'exercer, plus précisément, via les politiques de formation des salariés sur les risques professionnels. La présence d'un CHSCT multiplie par deux la probabilité qu'un salarié ait reçu une formation ou une information sur les risques professionnels au cours des douze derniers mois (Coutrot, 2009b). Enfin, la présence d'un CHSCT améliore fortement la qualité des politiques de prévention des risques professionnels (Coutrot, 2009b).

Nous pouvons noter que l'effet bénéfique de la présence d'un CHSCT sur la santé au travail se vérifie dans les établissements de petite taille $\left(\leq 50\right.$ salariés $\left.^{6}\right)$ qui n'ont pas l'obligation légale de disposer d'un CHSCT. Dans les établissements de moins 9 salariés dotés d'un CHSCT, $50 \%$ des salariés déclarent être en bonne santé au travail contre 39,3\% pour l'ensemble des salariés des établissements de cette taille ${ }^{7}$. La présence d'un CHSCT dans un établissement d'une aussi petite taille dénote une volonté d'amélioration de la santé au travail des salariés, de la part de la direction. Un « effet proximité » pourrait également jouer car, dans les établissements de petite taille, les membres des CHSCT ont toutes les chances d'être quotidiennement en contact avec l'ensemble des salariés. Les besoins des salariés, en termes de prévention des risques professionnels, seraient donc mieux connus et pris en compte. Par ailleurs, les analyses présentées dans le tableau 3b montrent que la présence d'un CHSCT est

\footnotetext{
${ }^{5}$ Résultats présentés par Coutrot (2009b) à partir de l'analyse des données de l'enquête Conditions de travail 2005, INSEE-DARES.

${ }^{6}$ Respectivement $10 \%$ et $20 \%$ des établissements de moins de 9 salariés et de 10 à 49 salariés ont un CHSCT, selon l'enquête SUMER 2003.

${ }^{7}$ De manière à avoir un indicateur de mesure synthétique de la santé au travail, nous avons sommé les scores pour nos quatre indicateurs de la santé au travail. Pour obtenir une échelle homogène, nous avons utilisé la formule suivante : santé au travail = quartile [Q1+Q2 + quartileQ3 + quartileQ4]. La cohérence interne de l'indicateur est acceptable $(\alpha=0,60)$. Nous avons ensuite dichotomisé à la moyenne la variable « santé au travail ». Les salariés qui ont un score inférieur ou égal à 8 sur une échelle qui va de 1 à 16 sont considérés comme ayant une mauvaise santé au travail.
} 
associée à une probabilité plus élevée de déclarer que son travail est mauvais pour sa santé ( $\beta$ $=0,067, p<0,1$ pour l'enquête SUMER 2003; $\beta=0,130, p<0,001$, tableau $1 \mathrm{~b}$; pour l'enquête SUMER 2010) ou stressant $(\beta=0,103, p<0,01$ pour l'enquête SUMER 2003, tableau 1b). Le CHSCT semble donc bien avoir des effets d'information et de prise de conscience concernant les relations entre travail et santé auprès des salariés, qui sont ensuite mieux à même d'inciter leurs représentants à agir au sein des CHSCT pour améliorer la qualité des politiques de prévention des risques professionnels. L'ensemble de ces analyses confirment que les mécanismes d'ordre cognitif (l'effet connaissance, cf. figure 1) expliquent les relations entre la présence d'un CHSCT et la santé au travail lorsqu'ils sont suivis de mécanismes d'ordre politique (l'effet pouvoir, cf. figure 1).

\subsection{La présence d'un CHSCT : un encouragement aux absences pour accident du travail ou un frein à la sous-déclaration?}

La relation entre la présence d'un CHSCT et les accidents du travail est peu documentée dans la littérature. Nous constatons qu'en présence d'un CHSCT, la durée des absences pour accident du travail est moins importante mais la fréquence des accidents du travail est plus élevée. Ce dernier résultat s'explique moins par le fait que la présence d'un CHSCT dans un établissement augmenterait la fréquence des accidents du travail que par le fait qu'il contribuerait à une diminution de la sous-déclaration des accidents du travail. L'implication des représentants salariés, membres du CHSCT, renforce la pression sur le management (Coutrot, 2009b) et pousse ainsi à faire reconnaître par la direction les accidents s'étant produits sur le lieu de travail, comme des accidents du travail. Ces mécanismes d'ordre politique (l'effet pouvoir, cf. figure 1) expliqueraient donc la relation positive entre la présence d'un CHSCT et la fréquence des absences pour accident du travail. Ceci rejoint les résultats observés par Walters et Nichols (2007) : la présence d'une IRP en charge de la santésécurité au travail augmenterait le taux d'accidents du travail. Selon ces auteurs, une 
explication possible serait que la présence de cette IRP inciterait les salariés à la déclaration de leurs accidents du travail.

\section{4 La présence d'un CHSCT : une durée plus courte des accidents du travail reliée à une politique de prévention des risques professionnels renforcée?}

Comme le relève Popma (2009), on ne peut s'en tenir à un constat d'une diminution de la gravité des accidents du travail en présence d'une instance représentative du personnel en charge des questions de santé au travail. Il faut aussi comprendre le canal par lequel s'effectue cette relation. Nos résultats démontrent que la présence d'un CHSCT, via des mécanismes d'ordres cognitif et politique (effets connaissance et pouvoir, cf. figure 1), est associée au déploiement d'une politique de prévention des risques professionnels qui est, à son tour, associée à une durée plus faible des absences pour accident du travail. Dans les deux CHSCT que nous avons observés (cf. notes d'observation dans le tableau 5), le temps consacré à l'analyse des causes des accidents du travail occupe une grande partie des réunions de CHSCT. Chaque accident donne lieu à une analyse approfondie des causes de l'accident du travail. Cette analyse entraîne la mise en place d'actions pour éviter sa survenue dans le futur.

Tableau 5. Notes d'observation portant sur l'analyse des accidents du travail dans le cadre d'une réunion des membres du CHSCT

\begin{tabular}{|c|c|}
\hline 20 janvier 2013, lors de la réunion du CHSCT 1 : & 30 janvier 2013, lors de la réunion du CHSCT 2 : \\
\hline $\begin{array}{l}\text { Directeur de l'établissement, président de la réunion } \\
\text { du CHSCT } 1 \text { : Un éboueur, intérimaire, a eu une main } \\
\text { coincée dans une manouvre de contournement. La } \\
\text { main a été coincée entre la benne et le poteau et a dû } \\
\text { être opérée. Il faudrait que les éboueurs descendent } \\
\text { du marchepied au moment des manceuvres. Un } \\
\text { souffleur (éboueur équipé d'une souffleuse permettant } \\
\text { de rassembler les feuilles mortes en un tas et de les } \\
\text { ramasser ensuite) a été percuté par un automobiliste } \\
\text { qui ne s'est pas arrêté... }\end{array}$ & $\begin{array}{l}\text { Directeur de l'établissement, président de la réunion } \\
\text { du CHSCT } 2 \text { : un agent s'est fait mal au dos. On a } \\
\text { revu l'organisation du travail. L'agent, victime d'un } \\
\text { accident du travail, n'a pas respecté les consignes de } \\
\text { gestes et postures, selon les dires de l'agent QHSE } \\
\text { Le chef d'atelier a fait une "causerie » pour rappeler } \\
\text { les consignes de gestes et postures... }\end{array}$ \\
\hline
\end{tabular}

Ces résultats convergent avec ceux d'une étude précédente menée dans le contexte néerlandais (Popma, 2009). Ce dernier auteur a montré que les principaux médiateurs dans la

\footnotetext{
${ }^{8}$ Technicien en charge de la qualité, de l'hygiène, de la sécurité et de l'environnement.
} 
relation entre la présence d'IRP en charge de la santé-sécurité au travail et la santé au travail sont : le recours à une expertise d'une administration publique spécialisée en santé et sécurité au travail; une politique managériale de prévention contre les nuisances et contre les pénibilités. Ces politiques limitent à leur tour la dégradation de la santé au travail causée par les expositions aux risques professionnels. Un autre facteur apparaît déterminant : le degré de mobilisation des acteurs membres du CHSCT et leur activisme de la connaissance (Hall et alii, 2006), consistant à une collecte stratégique de connaissances scientifiques, techniques, légales ainsi qu'à une utilisation tactique de ces mêmes connaissances (Hall et alii, 2006). L'activisme de la connaissance est une forme d'activisme politique conduisant à combiner les effets de connaissance et de pouvoir. Dans sa recherche comparative sur les modes de fonctionnement des CHSCT, Granaux (2012) croise ces deux variables, effet de connaissance et effet de pouvoir, qu'elle nomme « capital social » et « rapport de force » afin d'identifier quatre grands types de CHSCT : les CHSCT préventif, défensif, sortant et patronal. Notre observation directe de deux réunions de CHSCT (cf. notes d'observation dans le tableau 6) confirme ce constat en montrant que, d'une part, la qualité des politiques de prévention va dépendre de la plus ou moins grande sensibilité des représentants des salariés, membres du CHCST, à la santé au travail, d'autre part, l'activisme de connaissance des membres avec voix consultatives (médecin du travail, inspecteur du travail, représentant $\mathrm{du}$ service prévention de la Caisse Régionale d'Assurance Maladie d'Ile-de-France) sera déterminant pour la plus ou moins grande efficacité des CHSCT dans la mise en place d'une politique de prévention des risques professionnels. 
Tableau 6. Notes d'observation portant sur l'activisme de connaissance des participants à une réunion du CHSCT

\begin{tabular}{|c|c|}
\hline r 2013, lor & Г 2: \\
\hline $\begin{array}{l}\text { Les deux délégués syndicaux, membres du CHSCT, } \\
\text { ont une attitude inattendue : ils approuvent le } \\
\text { directeur, quand celui-ci dénonce le comportement de } \\
\text { l'éboueur absent " abusivement ", selon ses dires ; } \\
\text { quand celui-ci critique les éboueurs rechignant à } \\
\text { travailler lorsqu'il neige. Les deux délégués syndicaux } \\
\text { dénoncent les comportements " honteux ", selon eux, } \\
\text { des éboueurs qui volent le papier toilette, le gel } \\
\text { douche (comme s'ils se désolidarisaient de leurs } \\
\text { camarades en ne trouvant aucune explication à } \\
\text { avancer pour éclairer ces comportements : par } \\
\text { exemple, une contrainte financière forte pour certains } \\
\text { éboueurs, intérimaires). Ils en viennent à la fin de la } \\
\text { réunion à remercier les éboueurs pour leur " bon } \\
\text { comportement» (les éboueurs n'ont pas hésité, selon } \\
\text { eux, à collecter les ordures ménagères malgré les } \\
\text { risques pour leur sécurité, lors des tombées de neige). } \\
\text { Les deux délégués syndicaux, membres du CHSCT, } \\
\text { donnent l'impression de vouloir s'extraire de leur } \\
\text { groupe professionnel en calquant le discours de la } \\
\text { direction. Ils s'identifient totalement au discours de la } \\
\text { direction en se montrant obnubilés par l'activité } \\
\text { économique de l'agence. }\end{array}$ & $\begin{array}{l}\text { Nous remarquons que d'un CHSCT à l'autre, le rôle } \\
\text { du préventeur de la CRAMIF (Caisse Régionale } \\
\text { d'Assurance Maladie d'Ile-de-France) peut être } \\
\text { déterminant lorsqu'il y joue un rôle actif. C'est le cas } \\
\text { du représentant X de la CRAMIF, présent lors de la } \\
\text { réunion, qui exprime une demande de mise en place } \\
\text { d'une politique de prévention d'un risque } \\
\text { professionnel et veut suivre sa mise en place effective, } \\
\text { ce qui n'était pas le cas du représentant Y de la } \\
\text { CRAMIF lors de la réunion du CHSCT } 1 \text {. Son statut } \\
\text { est celui d'un membre invité mais son rôle est très } \\
\text { actif. Interventions révélatrices, selon nous, de } \\
\text { l'activisme du représentant X de la CRAMIF lors de } \\
\text { la réunion du CHSCT 2: "Ce serait bien que les } \\
\text { analyses des accidents du travail soient exposées en } \\
\text { CHSCT ", "Cela ne sert à rien de passer d'accident } \\
\text { en accident du travail sans préciser les causes! ", " } \\
\text { Avez-vous un plan de circulation au sein du site? Je } \\
\text { fais cette demande qu'il y ait un plan de circulation du } \\
\text { site pour éviter les collisions entre véhicules lourds ou } \\
\text { légers et les piétons. Vous pouvez vous appuyer sur un } \\
\text { document INRS sur l'établissement d'un plan de } \\
\text { circulation. Certes, toutes les demandes ne peuvent } \\
\text { être satisfaites. Mais il faut dresser un plan de } \\
\text { circulation du site. Ce serait intéressant de l'inscrire } \\
\text { à l'ordre du jour du prochain CHSCT ou du suivant». }\end{array}$ \\
\hline
\end{tabular}

Ces dernières analyses rejoignent des constats effectués par Legrand et Mias (2013) dans le cadre d'une recherche, effectuée dans 17 entreprises haut-normandes, portant sur l'action des CHSCT dans la prévention des risques cancérigènes: sans sensibilisation aux risques professionnels des représentants salariés des CHSCT (effet de connaissance), le risque est grand de voir le CSHCT défini comme l'instrument du service Hygiène, Sécurité et Environnement (HSE).

\section{Discussion}

Nous présentons les contributions théoriques, empiriques et méthodologiques de notre recherche. Nous développons ensuite les implications managériales. Enfin, nous soulignons les limites de notre recherche ainsi que les voies de recherche envisageables. 


\subsection{Contributions théoriques, empiriques et méthodologiques de la recherche}

Notre recherche apporte plusieurs contributions théoriques, empiriques et méthodologiques.

Tout d'abord, nous avons construit notre modèle de recherche en nous appuyant sur une théorie, peu utilisée en gestion des ressources humaines, la «politique de production» de Burawoy (1985). Ce cadre théorique nous aide à démontrer que la présence d'un CHSCT a des effets bénéfiques auprès des salariés au travers d'un renforcement du pouvoir des représentants des salariés (effet pouvoir), membres des CHSCT, leur permettant d'engager des actions collectives pour obliger la direction à adopter des mesures de prévention des risques professionnels. La présence d'un CHSCT contribue aussi à renforcer les connaissances des salariés sur les relations entre travail et santé (effet connaissance).

Ensuite, nos analyses économétriques corroborent un certain nombre d'études en montrant que la présence d'un CHSCT dans une organisation est associée à une meilleure santé au travail et à une durée plus faible des accidents du travail. Toutefois, notre étude souligne que cette relation ne vaut pas pour l'ensemble des indicateurs de mesure de la santé au travail ou des accidents du travail. Ainsi, la présence d'un CHSCT est associée à une meilleure santé au travail mesurée au travers de la réduction du risque pour un salarié de ne pas dormir à cause de son travail, et à une durée plus faible des absences pour accident du travail. Cependant, cet effet ne joue pas dans le sens attendu pour deux autres indicateurs de la santé au travail (travail stressant et lien subjectif entre travail et santé). Ces résultats inattendus pourraient s'expliquer par les effets d'information et de prise de conscience, auprès des salariés, sur les relations entre travail et santé, auxquels contribuerait le CHSCT. Parallèlement, nous constatons que la présence d'un CHSCT est associée à une fréquence plus élevée des absences pour accident du travail. La sous-déclaration des accidents du travail, une pratique avérée en France (Amossé et alii, 2012), pourrait expliquer cette corrélation positive, observée dans d'autres études internationales (Walters et Nichols, 2007). En outre, notre 
recherche montre que, dans le contexte français, contrairement aux deux autres études européennes portant sur le sujet, la présence d'un CHSCT est liée significativement à une probabilité plus faible d'être satisfait au travail. La présence d'un CHSCT semble en effet susciter chez les salariés des attentes non satisfaites sur l'amélioration des conditions de travail. Par ailleurs, notre recherche qualitative, par observation directe de deux réunions de CHSCT d'une même entreprise, souligne l'influence de l'implication des acteurs membres du CHSCT et de leur activisme de la connaissance (Hall et alii, 2006) sur la qualité de la politique de prévention des risques professionnels.

Enfin, notre étude illustre la pertinence d'une méthode mixte dans les recherches en gestion des ressources humaines. Or, comme le relèvent Aguinis et alii (2009), bien qu'ouvrant des perspectives nouvelles, ces méthodes sont, dans les faits, peu mobilisées dans le champ des études organisationnelles. L'observation directe non participante permet, à la fois, de donner une dimension qualitative à des régularités statistiques, parfois abstraites, mais aussi de fournir des déclinaisons contextualisées de tendances observées dans les analyses quantitatives. Dans notre cas, le choix d'un protocole (ou design) explicatif (Creswell et Plano Clark, 2011) permet d'affiner les résultats de l'analyse quantitative en comprenant mieux les mécanismes par lesquels la présence d'un CHSCT est associée à une durée plus faible des accidents du travail via une politique de prévention efficace.

\subsection{Implications managériales}

De ces résultats, nous déduisons des implications managériales. Trop souvent, les CHSCT sont assimilés à une «bête noire » ${ }^{9}$ par les directions d'entreprise. Or, nos résultats soulignent le rôle crucial des CHSCT pour les directions des ressources humaines souhaitant améliorer leur performance sociale. Les CHSCT peuvent être en effet un acteur important à mobiliser pour les services de gestion des ressources humaines dans le co-développement de politique

\footnotetext{
${ }^{9}$ Source : «En 30 ans, le CHSCT est devenu la " bête noire " des directions », Le Monde, 26 octobre 2012.
} 
de santé au travail et de prévention des risques professionnels. Pour les petits établissements de moins de 50 salariés, qui n'entrent pas dans le champ d'application de la législation française sur les CHSCT, la création en leur sein d'une IRP, chargée de la santé-sécurité au travail ou tout au moins d'un crédit d'heures pour les délégués du personnel qui exercent les missions du CHSCT dans les établissements de moins de 50 salariés, permettrait d'améliorer la santé au travail des salariés. Cette suggestion constitue une des propositions du rapport Verkindt (2014) sur les CHSCT. Pour les grands établissements, de 50 salariés et plus, la présence d'un CHSCT ne garantit pas qu'il soit actif. Pour qu'il le soit, il est nécessaire de donner, aux membres des IRP en charge de la santé-sécurité au travail, les moyens d'être à l'écoute de leurs collègues, par exemple, en augmentant les heures de délégation dont ils bénéficient et en leur proposant des formations sur la santé au travail et sur le pilotage social. Cette dernière proposition est aussi présente dans le rapport Verkindt (2014) sur les CHSCT.

Par ailleurs, les missions du CHSCT en termes de santé-sécurité au travail se révèlent être de plus en plus décisives pour l'image sociale de l'entreprise puisque les problèmes de santé au travail sont au centre de l'actualité. Les problèmes de santé au travail sont en effet une préoccupation majeure dans les pays développés. Le rôle du CHSCT est donc amené à s'accroître. La synthèse des accords signés par les entreprises de plus de 1000 salariés sur la prévention des risques psychosociaux ${ }^{10}$ souligne la présence de dispositions prévoyant l'information et la consultation du CHSCT dans $75 \%$ des accords. Des accords-cadres internationaux (ACI), conclus avant tout dans une perspective d'amélioration de l'image sociale de l'entreprise et souvent limités à un rappel des Droits fondamentaux au travail issus des Conventions de base de l'OIT (Barreau et Arnal, 2010), portent désormais sur la thématique de la santé-sécurité au travail. C'est le cas des ACI conclus par trois groupes: Daimler-Chrisler (2006), Arcelor-Mittal (2008) et Danone (2011).

\footnotetext{
${ }^{10}$ Source : Synthèse des accords signés par les entreprises de plus de 1000 salariés sur la prévention des risques psychosociaux, Rapport de la Direction Générale du Travail, avril 2011.
} 
Enfin, le domaine d'intervention du CHSCT pourrait s'accroitre dans les années à venir et pourrait englober l'organisation du travail. Ainsi, un arrêt du 5 mars 2008 ( ${ }^{\circ}$ 06-45888, Snecma) de la chambre sociale de la Cour de Cassation incitera de plus en plus les CHSCT à remettre en cause l'organisation du travail, en s'appuyant sur l'obligation de résultat de l'employeur qui concerne l'ensemble des dispositions de l'article L.4121-1 du Code du travail $^{11}$. Dans son arrêt du 5 mars $2008\left(n^{\circ}\right.$ 06-45888, Snecma), la chambre sociale de la Cour de Cassation a annulé une réorganisation du travail, mise en œuvre malgré un avis négatif du CHSCT, après expertise, en considérant que «l'obligation de sécurité de résultat » de l'employeur lui interdisait «dans l'exercice de son pouvoir de direction, de prendre des mesures qui auraient pour objet ou pour effet de compromettre la santé et la sécurité des salariés » (arrêt n 06-45888, Snecma, cité par Verkindt, 2008). En d'autres termes, l'impératif de santé prime le pouvoir de direction, lequel peut être remis en question s'il est exercé de telle sorte que cette santé soit compromise (Verkindt, 2008). Sous l'effet de cette jurisprudence, le CHSCT connaît une véritable mutation : «la montée en puissance de la notion de santé conjuguée à la prise de conscience des facteurs sociaux et organisationnels des risques psychosociaux contribuent à ce que le CHSCT puisse «s'inviter» dans les domaines relevant des prérogatives patronales » (Del Sol, 2013, p. 6).

\subsection{Limites et voies futures de recherche}

Bien que la présente recherche contribue à enrichir la littérature sur le rôle des IRP en matière de santé-sécurité au travail, elle comporte des limites. Premièrement, les données sont évaluées en coupe instantanée. Nous ne pouvons pas tirer de conclusions fermes sur les inférences causales entre la présence d'un CHSCT et les variables à expliquer. Seule une étude longitudinale pourrait donner des conclusions fermes sur le sens de la causalité. De

\footnotetext{
11 Selon l'article L.4121-1 du Code du travail, l'employeur prend les mesures nécessaires pour assurer la sécurité et protéger la santé physique et mentale des travailleurs : actions de prévention des risques professionnels, actions d'information et de formation, mise en place d'une organisation et de moyens adaptés.
} 
même, il aurait été opportun, si les enquêtes SUMER 2003 et 2010 nous l'avaient permis, d'utiliser des analyses multi-niveaux. En effet, la présence d'un CHSCT est une caractéristique de l'établissement, alors que le bien-être caractérise des salariés particuliers de cet établissement non forcément représentatifs de la situation moyenne de l'établissement. Toutefois, dans le prolongement des remarques de Coutrot (2009b), cette différence de niveaux d'analyse entre la variable à expliquer et la variable explicative crée un risque de «bruit» statistique qui pourrait induire une sous-estimation (et non une sur-estimation) de l'intensité des liens entre la présence des CHSCT et le bien-être des salariés. Les coefficients de régression estimés dans notre recherche doivent donc être considérés «comme les bornes inférieures de l'intensité des liens mesurés» (Coutrot, 2009b, p. 30). Deuxièmement, le nombre limité d'indicateurs de mesure du bien-être présents dans les enquêtes SUMER 2003 et 2010 ne permet pas d'opérationnaliser de manière précise la santé au travail. Des études complémentaires, offrant une palette plus large d'indicateurs, seraient nécessaires. Troisièmement, nous n'avons pas pu tester les effets médiateurs des politiques de prévention dans la relation entre la présence d'un CHSCT et la santé au travail, faute de variables sur ce sujet présentes dans les enquêtes SUMER 2003 et 2010. Cette recherche pourrait donc être prolongée en menant des études de cas longitudinales, à partir d'entretiens et d'observations, sur les effets à moyen et long terme de la présence d'une IRP en charge de la santé-sécurité au travail sur la santé au travail via les politiques de prévention mises en place sous l'influence d'un CHSCT.

\section{Conclusion}

Notre recherche constitue une première étude exploratoire sur un sujet encore très peu traité. Peu d'analyses économétriques ont en effet exploré le rôle des IRP en charge de la santésécurité au travail sur le bien-être des salariés (Bryce et Manga, 1985 ; Walters et Nichols, 2007). Notre étude souligne l'association significative entre la présence d'un CHSCT et le 
bien-être des salariés et une moindre durée des accidents du travail. En outre, nos analyses démontrent que la présence d'un CHSCT, via des mécanismes d'ordres cognitif et politique, est associée à la mise en place d'une politique de prévention des risques professionnels qui est, à son tour, associée à durée plus faible des absences pour accident du travail. Enfin, notre étude qualitative souligne l'importance de l'activisme des connaissances des acteurs membres du CHSCT dans la plus ou moins grande efficacité des politiques de prévention initiées par cette instance. Nos analyses nous conduisent à proposer que les directions des ressources humaines associent les IRP en charge de la santé-sécurité au travail à l'élaboration de politiques de santé au travail et de prévention des risques professionnels.

\section{Bibliographie}

AGUINIS, H., PIERCE, C.A., BOSCO, F.A., MUSLIN, I.S. (2009), «First decade of Organizational Research Methods. Trends in design, measurement and data-analysis topics », Organizational Research Methods, vol. 12, $\mathrm{n}^{\circ}$ 1, p. 69-112.

AMOSSE, T., DAUBAS-LETOURNEUX, V., LE ROY, F., MESLIN, K., BARRAGAN, K. (2012), « Les accidents du travail et problèmes de santé liés au travail dans l'enquête SIP. Invisibilités et inscriptions dans les trajectoires professionnelles », Rapport de recherche du CEE, $\mathrm{n}^{\circ} 76$.

ARBORIO, A.M., FOURNIER, P. (1999), L'enquête et ses méthodes : l'observation directe, Paris, Nathan.

AUROUX, J. (2012), « Son droit d'expression. Entretien avec Jean Auroux », NVO. La Nouvelle Vie Ouvrière, H.S n ${ }^{\circ}$, octobre 2012, p. 22-25.

BARON, R.M., KENNY, D.A. (1986), « The moderator-mediator variable distinction in social psychological research: conceptual, strategic, and statistical considerations », Journal of Personality and Social Psychology, vol. 51, n ${ }^{\circ}$, p. 1173-1182.

BARREAU, J., ARNAL, J. (2011), « Effects of Financialization on Restructuring and Sustainable Development Policy: the Accor group Case », in SUN W., LOUCHE C. and PEREZ R. (ed.), Finance and Sustainability: Towards a New Paradigm? A Post-Crisis Agenda, Bingley, Emerald books, 2011, p. 249-272.

BIETRY, F., CREUSIER, J. (2013), «Proposition d'une échelle de mesure positive du bien-être au travail (EPBET) », Revue de Gestion des Ressources Humaines, vol. 87, p. 23-41.

BÖCKERMAN, P., ILAMAKUNNAS, P. (2009), « Job Disamenities, Job Satisfaction, Quit Intentions, and Actual Separations: Putting the Pieces Together », Industrial Relations, vol. $48, \mathrm{n}^{\circ} 1$, p. 73-96.

BÖCKERMAN, P., BRYSON, A. ILMAKUNAS, P. (2012), «Does high invovlment management improve worker wellbeing? », Journal of Economic Behavior \& Organization, vol. 84, n 6, p. 660680.

BOUVILLE, G. (2009), «L'influence de l'organisation et des conditions de travail sur l'absentéisme. Analyse quantitative et étude de cas ", Thèse de Doctorat en Sciences de Gestion, Université de Rennes 1. 
BREGIER, G., BREGIER, V. (2015), Le CHSCT en pratique, Eyrolles, Paris, 2015.

BRYCE, G.K.., MANGA, P. (1985), "The Effectiveness of Health and Safety Committees », Relations Industrielles/Industrial Relations, vol. 40, $\mathrm{n}^{\circ} 2$, p. 257-283.

BUE, J., COUTROT, T., GUIGNON, N., SANDRET, N. (2008), « Les facteurs de risques psychosociaux au travail. Une approche quantitative par l'enquête SUMER », Revue française des Affaires sociales, vol. 2-3, p. 45-70.

BURAWOY, M. (1985), The Politics of Production. Factory Regimes Under Capitalism and Socialism, London, Verso.

COUTROT, T. (2009a), « Health and safety committees in France: an empirical analysis », in Walters, D. \& Nichols, T. (2009), Workplace Health and Safety, London, Palgrave MacMillan, p. 50-72.

COUTROT, T. (2009b), «Le rôle des comités d'hygiène, de sécurité et des conditions de travail en France : une analyse empirique », Travail et Emploi, vol. 117, p. 25-38.

CRESWELl, J.W., PLANO CLARK, V.L. (2011), Designing and Conducting Mixed Methods Research, $2^{\text {nd }}$ Ed., Thousand Oaks, CA: Sage.

DEL SOL, M. (2013), «Les décisions et pratiques managériales à l'épreuve du droit à la santé au travail », Revue de droit sanitaire et social, vol. 5, p. 1-16.

FERRIE, J.E., KIVIMÄKI, M., HEAD, J., SHIPLEY, M.J., VAHTERA, J., MARMOT, M.G. (2005), «A comparison of self-reported sickness absence with absences recorded in employers' registers: evidence from the Whitehall II study », Occupational and Environmental Medicine, vol. 62, $\mathrm{n}^{\circ} 2$, p.74-79.

FOSTER, E. M. (1997), «Instrumental variables for logistic regression: An illustration », Social Science Research, vol. 26, n 4, 487-504.

FREEMAN, R., MEDOFF, J. (1984), What Do Unions Do?, New York, Basic Books.

GRANAUX, S. (2012), «Les CHSCT aux prises avec l'évaluation des risques du travail dans un contexte d'individuation de la relation salariale et de déclin des représentations collectives : quatre cas de figure et deux exemples de CHSCT préventifs », Communication aux XIIIème Journées internationales de sociologie du travail, Université libre de Bruxelles, 25-27 janvier.

HALL, A., FORREST, A., SEARS, A., CARLAN, N. (2006), « Making a difference. Knowledge activism and worker representation in joint OHS committee», Relations Industrielles/Indutrial Relations, vol. 61, $\mathrm{n}^{\circ} 3$, p. 408-436.

KAPLAN, G.A., GOLDBERG, D.E., EVERSON, S.A., COHEN, R.D., SALONEN, R., TUOMILEHTO, J., SALONENE, J. (1996), «Perceived health status and morbidity and mortality: evidence from the Kuopio ischaemic heart disease risk factor study », International Journal of Epidemiology, vol. 25, $\mathrm{n}^{\circ}$ 2, p. 259-265.

LEGRAND, E., MIAS, A. (2013), «Zones d'ombre dans la prévention des risques cancérogènes », Sociologies pratiques, vol. 26, p. 71-83.

LEROUGE, L., VERKINDT, P. Y. (2015), « Sauvegarder et renforcer le CHSCT : un enjeu majeur de santé au travail », Droit social, vol. 4 - avril 2015, p. 365-366.

LEYMANN, H. (1996), « The content and development of mobbing at work », European Journal of Work and Organizational Psychology, vol. 5, $\mathrm{n}^{\circ}$ 2, p.165-184.

LUCHMAN J.N., GONZALEZ-MORALES M.G. (2013), «Demands, control, and support: ametaanalytic review of work characteristics interrelationships », Journal of Occupantional Health Psychology, vol. 18, $\mathrm{n}^{\circ}$ 1, p. 37-52.

MBENGUE, A., VANDANGEON-DERUMEZ, I. (2003), «Analyse causale et modélisation », in Thiétart, R.A. (coord.), Méthodes de recherche en management, Paris, Dunod, p. 335-372. 
MOREAU, M. A. (2002), « Pour une politique de santé dans l'entreprise », Droit Social, vol. 9/10, p. 817-827.

NICHOLS, T., WALTERS, D. AND TASIRAN, A.C. (2007), « Trade Unions, Institutional Mediation and Industrial Safety: Evidence from the UK », The Journal of Industrial Relations, vol. 49, $\mathrm{n}^{\circ}$ 2, $\mathrm{p}$. 211-225.

POPMA, J.R. (2009), «Does worker participation improve health and safety? Findings from the Netherlands », Policy and Practice in Health and Safety, vol. 7, n 1, p. 33-51.

PREACHER, K.J., HAYES, A.F. (2004), «SPSS and SAS procedures for estimating indirect effects in simple mediation models», Behavior Research Methods, Instruments, \& Computers, vol. 36, $\mathrm{n}^{\circ}$ 4, p. 717-731.

RELIHAN E., GLYNN S., DALY D., SILKIC B. (2009), «Measuring and benchmarking safety culture: application of the safety attitudes questionnaire to an acute medical admission unit », Irish Journal of Medical Science, vol. 178, n ${ }^{\circ}$ 4, p. 433-439.

RYAN, R. M., DECI, E. L. (2001), « On happiness and human potentials: A review of research on hedonic and eudaimonic well-being », Annual Review of Psychology, vol. 52, n 1, p. 141-166.

SINGH-MANOUX, A., MARTIKAINEN, P., FERRIE, J., ZINS, M., MARMOT, M., GOLDBERG, M. (2006), «What does self rated health measure? Results from the British Whitehall II and French Gazel cohort studies », Journal of Epidemiology and Community Health, vol. 60, n 4, p. 364-372.

SPARKS, K., FARAGHER, B., COOPER, C. (2010), "Well-being and occupational health in the 21st century workplace », Journal of Occupational and Organizational Psychology, vol. 74, $\mathrm{n}^{\circ}$ 4, p. 489-509.

SUN W., LOUCHE C. and PEREZ R. (2011), Finance and Sustainability: Towards a New Paradigm? A Post-Crisis Agenda, Bingley, Emerald books.

TENENHAUS, M. (2007), Statistique. Méthodes pour décrire, expliquer et prévoir, Paris, Dunod.

THIETART, R.A. (2003), Méthodes de recherche en management, Paris, Dunod.

TIMS, M., BAKKER, A.B., DERKS, D. (2013), «The impact of job crafting on job demands, job resources, and well-being », Journal of Occupational Health Psychology, vol. 18, n 2, p. 230-240.

TORP, S., MOEN, B.E. (2006), « The effects of occupational health and safety management on work environment and health: A prospective study », Applied Ergonomics, vol. 37, n 6, p. 775-783.

VERKINDT, P.Y. (2008), «Santé au travail vs pouvoir de direction. Un retour de la théorie institutionnelle de l'entreprise ? », Droit Social, vol. 5, p. 519-520.

VERKINDT, P.Y. (2014), «Le C.H.S.C.T au milieu du gué », Rapport pour le Ministre du travail, de l'emploi, de la formation professionnelle et du dialogue social.

VOSS, M., STARK, S., ALFREDSSON, L., VINGARD, E., JOSEPHSON, M. (2008), « Comparisons of self-reported and register data on sickness absence among public employees in Sweden», Occupational and Environmental Medicine, vol. 65, $\mathrm{n}^{\circ}$ 1, p. 61-67.

WACHEUX, F. (1996), Méthodes qualitatives et recherche en gestion, Paris, Economica.

WALTERS, D., FRICK K. (2000), « Worker participation and the management of health and safety: reinforcing or conflicting strategies? », in Systematic Occupational Health and Safety Management: Perspectives on an International Development, Frick, K., Jensen, P.L., Quinlan, M., Wilthagen, T., New York, Pergamon.

WALTERS, D., NICHOLS, T. (2009), Workplace Health and Safety, New York, Palgrave MacMillan.

WALTERS, D., NICHOLS, T. (2007), Worker Representation and Workplace Health and Safety, New York, Palgrave MacMillan. 
WARNOUS, J.P, REICHERS, A.E., HUDY, M.J. (1997), «Overall job satisfaction: How good are single-item measures? », Journal of Applied Psychology, vol. 82, n 2, p. 247-252.

\section{Annexe}

\section{Mesures des variables de contrôle}

Les variables de contrôle de notre étude sont les suivantes :

Variables «structurelles »
Taille de l'établissement, le secteur d'activité, sexe, âge, ancienneté, catégorie socio-professionnelle (CSP), statut de l'emploi
Conditions de travail

Postures douloureuses, nuisances thermiques et sonores, tensions avec le public, harcèlement moral

Les postures douloureuses est une variable dichotomique mesurée par l'item suivant : «Avez-vous été exposé(e) à une posture douloureuse suivante : déplacement à pied dans le travail, mouvements répétitifs, position debout, position à genoux, maintien de bras en l'air, au moins 20 heures lors de la semaine précédente?».

Les nuisances thermiques est une variable dichotomique mesurée par l'item suivant : «Avez-vous été exposé(e) à une nuisance thermique suivante : travail à une température $>24^{\circ} \mathrm{C},<-15^{\circ} \mathrm{C}$, travail en milieu humide ou travail à l'extérieur, au moins 20 heures lors de la semaine précédente?».

Les nuisances sonores est une variable dichotomique mesurée par l'item suivant : "Avez-vous été exposé(e) au moins à une pollution sonore $: \geq 85$ décibels, des chocs, des impulsions, autre bruit gênant, au moins 20 heures au sein de la semaine précédente?».

Les tensions avec le public a été mesurée par l'item suivant : «Vivez-vous des situations de tension dans vos rapports avec le public? ». Les participants répondaient sur une échelle à 4 points.

Le harcèlement moral est une variable dichotomique mesurée par l'item suivant : «Avez-vous été exposé systématiquement à une forme de situations difficiles suivantes (parmi 10 formes de harcèlement proposées par Leymann (1996))?». 